\title{
SO(3) family symmetry and axions
}

\author{
Mario Reig, ${ }^{1, *}$ José W. F. Valle, ${ }^{1, \dagger}$ and Frank Wilczek ${ }^{2,3,4,5, \$}$ \\ ${ }^{1}$ AHEP Group, Institut de Física Corpuscular, C.S.I.C./Universitat de València, \\ Parc Científic de Paterna. C/ Catedrático José Beltrán, 2 E-46980 Paterna (Valencia), Spain \\ ${ }^{2}$ Center for Theoretical Physics, Massachusetts Institute of Technology, \\ Cambridge, Massachusetts 02139, USA \\ ${ }^{3}$ Tsung-Dao Lee Institute and Wilczek Quantum Center, Shanghai 200240, China \\ ${ }^{4}$ Department of Physics, Stockholm University, Stockholm SE-106 91, Sweden \\ ${ }^{5}$ Department of Physics and Origins Project, Arizona State University, Tempe, Arizona 25287, USA
}

(Received 4 June 2018; published 13 November 2018)

\begin{abstract}
Motivated by the idea of comprehensive unification, we study a gauged $S O(3)$ flavor extension of the extended Standard Model, including right-handed neutrinos and a Peccei-Quinn symmetry with simple charge assignments. The model accommodates the observed fermion masses and mixings and yields a characteristic, successful relation among them. The Peccei-Quinn symmetry is an essential ingredient.
\end{abstract}

DOI: $10.1103 /$ PhysRevD.98.095008

\section{INTRODUCTION AND MOTIVATION}

For all its success, the Standard Model has many loose ends and shortcomings. It leaves unexplained the threefold family replication, the observed pattern of quark and lepton masses and mixings, and the lack of $C P$ violation in the strong interaction [1-3]. It does not account for the cosmological dark matter [4], and in its minimal form it leaves neutrinos massless [5].

In addressing those questions, it is natural to consider extending the ideas of gauge symmetry and its spontaneous breaking beyond their established, central role in the Standard Model. $S U(3)$ and $S O(3)$ suggest themselves as candidate symmetries for family unification, since they support irreducible triplet representations. (Discrete symmetries can also be gauged, and quantum gravity might require that they are [6,7].) $S O(3)$ is particularly attractive, since it arises naturally in the context of comprehensive unification, which brings together forces and flavor [8-12].

In this paper we explore an $S O(3)$ family symmetry model inspired by comprehensive unification. Within a reasonably economical model, several appealing features emerge:

\footnotetext{
*mario.reig@ific.uv.es

†valle@ific.uv.es

¥wilczek@mit.edu
}

Published by the American Physical Society under the terms of the Creative Commons Attribution 4.0 International license. Further distribution of this work must maintain attribution to the author(s) and the published article's title, journal citation, and DOI. Funded by SCOAP. (i) a Peccei-Quinn symmetry, leading to axions, which is both natural and helpful to ensure correct mass relations,

(ii) extreme fine-tuning is not required,

(iii) a characteristic "golden" formula relating quark and lepton masses, given in Eq. (10) [13-15],

(iv) a successful explanatory framework for the Cabibbo-Kobayashi-Maskawa (CKM) matrix, with two predictions, Eqs. (12) and (13), and

(v) a conventional seesaw mechanism [8] [16-21] for neutrino mass generation at the Peccei-Quinn (PQ) scale, supplemented by a connection between lepton number and PQ breaking, which relates the axion and neutrino mass scales, Eq. (18).

\section{MODEL CONSTRUCTION}

\section{A. $\mathbf{S O}(3)$ as family symmetry}

Discrete [13-15] and continuous [22,23] horizontal flavor symmetries have been used extensively in model building. Many options have been considered. It is interesting to consider, as a source of guidance, their possible deeper origin. In [12] we revived the idea of comprehensive unification, merging gauge and family symmetry. The striking fact that one can accommodate the observed fermions into a single irreducible spinor multiplet of large orthogonal groups encourages such ideas, which have a long history (see, e.g., [9]). On the face of it, however, they contain too many families, and also an equal number of wrong-chirality "antifamilies" (but no other exotics). We suggested that extraneous families are confined at a high scale $\gtrsim 10 \mathrm{TeV}$, while the antifamilies were removed through an orbifold construction. 
TABLE I. Particle content and transformation properties under the $\mathrm{SU}(3)_{\mathrm{c}} \otimes \mathrm{SU}(2)_{\mathrm{L}} \otimes \mathrm{U}(1)_{\mathrm{Y}}$ and flavor $S O(3)$ gauge groups. The vacuum expectation values (VEVs) of $\mathrm{SU}(3)_{\mathrm{c}} \otimes \mathrm{SU}(2)_{\mathrm{L}} \otimes$ $\mathrm{U}(1)_{\mathrm{Y}}$ singlets $\sigma$ and $\rho$ break $U(1)_{\mathrm{PQ}}$ and lepton number, generating Majorana neutrino masses.

\begin{tabular}{lcccccccccccc}
\hline \hline & $q_{L}$ & $u_{R}$ & $d_{R}$ & $l_{L}$ & $e_{R}$ & $\nu_{R}$ & $\Phi^{u}$ & $\Phi^{d}$ & $\Psi^{u}$ & $\Psi^{d}$ & $\sigma$ & $\rho$ \\
\hline $\mathrm{SU}(3)_{\mathrm{c}}$ & $\mathbf{3}$ & $\mathbf{3}$ & $\mathbf{3}$ & $\mathbf{1}$ & $\mathbf{1}$ & $\mathbf{1}$ & $\mathbf{1}$ & $\mathbf{1}$ & $\mathbf{1}$ & $\mathbf{1}$ & $\mathbf{1}$ & $\mathbf{1}$ \\
$\mathrm{SU}(2)_{\mathrm{L}}$ & $\mathbf{2}$ & $\mathbf{1}$ & $\mathbf{1}$ & $\mathbf{2}$ & $\mathbf{1}$ & $\mathbf{1}$ & $\mathbf{2}$ & $\mathbf{2}$ & $\mathbf{2}$ & $\mathbf{2}$ & $\mathbf{1}$ & $\mathbf{1}$ \\
$\mathrm{U}(1)_{\mathrm{Y}}$ & $\frac{1}{6}$ & $\frac{2}{3}$ & $-\frac{1}{3}$ & $-\frac{1}{2}$ & -1 & 0 & $-\frac{1}{2}$ & $\frac{1}{2}$ & $-\frac{1}{2}$ & $\frac{1}{2}$ & 0 & 0 \\
$\mathrm{SO}(3)_{\mathrm{F}}$ & $\mathbf{3}$ & $\mathbf{3}$ & $\mathbf{3}$ & $\mathbf{3}$ & $\mathbf{3}$ & $\mathbf{3}$ & $\mathbf{5}$ & $\mathbf{5}$ & $\mathbf{3}$ & $\mathbf{3}$ & $\mathbf{5}$ & $\mathbf{1}$ \\
$\mathrm{U}(1)_{\mathrm{PQ}}$ & 1 & -1 & -1 & 1 & -1 & -1 & 2 & 2 & 2 & 2 & 2 & 2 \\
\hline \hline
\end{tabular}

More specifically, the breaking scheme $S O(18) \rightarrow$ $S O(10) \times S O(5) \times S O(3)$ (see [8]) allows for the standard, attractive, $S O(10)$ gauge unification, together with a hypercolor $S O(5)$ which confines 5 families (leaving 3) and an $S O(3)$ family symmetry group. This motivates consideration of $S O(3)$ as a family unification group.

More generally, $S O(3)$ family symmetry is more easily compatible with gauge unification than is $S U(3)$ family symmetry. In the usual $S U(5)$ and $S O(10)$ theories one embeds the Standard Model particle content in the anomaly free sets of representations: $3 \times(\overline{\mathbf{5}}+\mathbf{1 0})$ for $S U(5)$ and $3 \times \mathbf{1 6}$ for $S O(10)$. Assigning these representations as $S U$ (3) triplets generally leads to anomalies. For example, in the $S O(10) \times S U(3)$ theory the standard $(\mathbf{1 6}, \mathbf{3})$ combination has an $\left[S U(3)_{F}\right]^{3}$ anomaly.

\section{B. Field content}

We now develop a consistent flavor extension of the Standard Model in which the gauge symmetry is enlarged by adding the local $S O(3)_{F}$ family symmetry [22]. In addition to Standard Model particles, the model has an enlarged scalar sector and right-handed neutrinos. This minimal extension is enough to accommodate fermion masses and mixings without fine-tuning of parameters and the other features mentioned earlier.

The field content of our model is displayed in Table I. Especially noteworthy are the Peccei-Quinn charge assignments. ${ }^{1}$ They arise from a transformation that commutes with $S O(10)$ : all the fermion fields which occur in the $S O(10)$ spinor have the same PQ charge. It also commutes with $S O(3)_{F}$.

\section{Symmetry breaking}

In our model symmetry breaking proceeds through the following set of scalar fields:

\footnotetext{
${ }^{1}$ Recently, an alternative framework with flavor-dependent Peccei-Quinn charges has been proposed in $[24,25]$. Our $U(1)_{\mathrm{PQ}}$ symmetry is related to flavor in a rather different way, through the $S O(3)$ family symmetry.
}

$$
\begin{aligned}
\Psi^{u} & \sim(\mathbf{1}, \mathbf{2},-1 / 2, \mathbf{3}), \\
\Psi^{d} & \sim(\mathbf{1}, \mathbf{2}, 1 / 2, \mathbf{3}), \\
\Phi^{u} & \sim(\mathbf{1}, \mathbf{2},-1 / 2, \mathbf{5}), \\
\Phi^{d} & \sim(\mathbf{1}, \mathbf{2}, 1 / 2, \mathbf{5}), \\
\sigma & \sim(\mathbf{1}, \mathbf{1}, 0, \mathbf{5}), \\
\rho & \sim(\mathbf{1}, \mathbf{1}, 0, \mathbf{1}) .
\end{aligned}
$$

There are two $\mathrm{SU}(3)_{\mathrm{c}} \otimes \mathrm{SU}(2)_{\mathrm{L}} \otimes \mathrm{U}(1)_{\mathrm{Y}}$ singlet scalars, $\sigma \sim(\mathbf{1}, \mathbf{1}, 0, \mathbf{5})$ and $\rho \sim(\mathbf{1}, \mathbf{1}, 0, \mathbf{1})$. All of these fields will acquire nontrivial vacuum expectation values.

Both $S O(3)$ singlets as well as the quintuplet carry nontrivial PQ charges. Therefore, the spontaneous breaking of the Peccei-Quinn symmetry is triggered by their large VEVs. On the other hand, the $S O(3)$ family symmetry breaking is associated to the $\mathrm{VEV}$ of the $\mathrm{SU}(3)_{\mathrm{c}} \otimes$ $\mathrm{SU}(2)_{\mathrm{L}} \otimes \mathrm{U}(1)_{\mathrm{Y}}$ singlet scalar $\sigma$;

$\langle\sigma\rangle=v_{\sigma} \operatorname{diag}(0,1,-1) \quad$ and $\quad\langle\rho\rangle=v_{\rho} \operatorname{diag}(1,1,1)$.

As we will see later, both VEVs play a key role in breaking lepton number, generating Majorana neutrino mass, and accounting for the large neutrino mixing angles observed in neutrino oscillations.

In order to break the electroweak symmetry we assume VEVs for the $\mathrm{SU}(2)_{\mathrm{L}}$ scalar doublets, i.e., $\Phi^{u}$ and $\Phi^{d}$, transforming as $S O(3)$ quintuplets, as well as $\Psi^{u}$ and $\Psi^{d}$, transforming as $S O(3)$ triplets. We assume the following pattern for the VEVs:

$$
\begin{aligned}
& \left\langle\Phi^{u, d}\right\rangle=\left(\begin{array}{ccc}
0 & & \\
& -k^{u, d} & \epsilon_{1}^{u, d} \\
& \epsilon_{1}^{u, d} & k^{u, d}
\end{array}\right), \\
& \left\langle\Psi^{u, d}\right\rangle=\left(\begin{array}{c}
v^{u, d} \\
0 \\
\epsilon_{2}^{u, d}
\end{array}\right),
\end{aligned}
$$

where the small parameters $\epsilon_{i}$ denote a perturbation with respect to the simplest alignments $\operatorname{diag}(0,-1,1)$ and $(1,0,0)$. This symmetry breaking pattern minimizes the Higgs potential [22], and provides a good description of the observed fermion mass hierarchy; see below.

An important feature of the model is the existence of a spontaneously broken U(1) global PQ symmetry. For definiteness, we fix the PQ quantum numbers as given in Table I. The VEVs of $\mathrm{SU}(3)_{\mathrm{c}} \otimes \mathrm{SU}(2)_{\mathrm{L}} \otimes \mathrm{U}(1)_{\mathrm{Y}}$ singlets $\sigma$ and $\rho$ break $U(1)_{\mathrm{PQ}}$ as well as lepton number. The alignment of the associated Nambu-Goldstone boson $G$ is

$$
G \approx \frac{1}{\left(v_{\sigma}^{2}+v_{\rho}^{2}\right)^{1 / 2}}\left(v_{\sigma} \sigma^{I}+v_{\rho} \rho^{I}+\ldots\right)
$$


where $\rho^{I}$ etc. denote the imaginary parts of scalars and ... denotes components along the isodoublet scalars $\Psi^{u I}, \Psi^{d I}$, $\Phi^{u I}, \Phi^{d I}$, weighted by their VEVs and times their PQ charges. Notice that, through these projections, $G$ will couple directly to quarks and leptons at the tree level. These couplings are suppressed linearly by the PQ-breaking scale $\left(v_{\sigma}^{2}+v_{\rho}^{2}\right)^{1 / 2}$. Thus we arrive at a model of the DineFischler-Srednicki-Zhitnitsky type [26] including coupling to neutrinos.

\section{III. “GOLDEN FORMULA" FOR QUARKS AND LEPTON MASSES}

Given the $S O(3)$ multiplication rules, $\mathbf{3} \times \mathbf{3}=\mathbf{1}+\mathbf{3}+\mathbf{5}$, one can use the vector (triplet) and the two-index symmetric traceless tensor (quintuplet) representations to build the following invariant Yukawa Lagrangian:

$$
\begin{aligned}
\mathcal{L}= & \bar{q}_{L}\left(y_{1} \Psi^{u}+y_{2} \Phi^{u}\right) u_{R}+\bar{q}_{L}\left(y_{3} \Psi^{d}+y_{4} \Phi^{d}\right) d_{R} \\
& +\bar{l}_{L}\left(y_{5} \Psi^{d}+y_{6} \Phi^{d}\right) e_{R}+\text { H.c. }
\end{aligned}
$$

Note that the "duplicated" scalar sector, with two scalar doublets selectively coupled to up-type/down-type fermions, does not imply a nonminimal low-energy Higgs sector, as we shall discuss further below.

After electroweak breaking, Eq. (5) leads to the quark mass matrices

$$
\begin{aligned}
& M^{u}=\left(\begin{array}{ccc}
0 & y_{1} \epsilon_{2}^{u} & 0 \\
-y_{1} \epsilon_{2}^{u} & -y_{2} k^{u} & y_{1} v^{u}+y_{2} \epsilon_{1}^{u} \\
0 & -y_{1} v^{u}+y_{2} \epsilon_{1}^{u} & y_{2} k^{u}
\end{array}\right), \\
& M^{d}=\left(\begin{array}{ccc}
0 & y_{3} \epsilon_{2}^{d} & 0 \\
-y_{3} \epsilon_{2}^{d} & -y_{4} k^{d} & y_{3} v^{d}+y_{4} \epsilon_{1}^{d} \\
0 & -y_{3} v^{d}+y_{4} \epsilon_{1}^{d} & y_{4} k^{d}
\end{array}\right),
\end{aligned}
$$

and for the charged leptons

$$
M^{e}=\left(\begin{array}{ccc}
0 & y_{5} \epsilon_{2}^{d} & 0 \\
-y_{5} \epsilon_{2}^{d} & -y_{6} k^{d} & y_{5} v^{d}+y_{6} \epsilon_{1}^{d} \\
0 & -y_{5} v^{d}+y_{6} \epsilon_{1}^{d} & y_{6} k^{d}
\end{array}\right) \text {, }
$$

where we take into account the VEV alignment patterns of the $S O(3)$ triplet and quintuplet scalars, respectively.

These matrices allow a good description of the charged fermion masses. Indeed, neglecting the $\epsilon_{i}$ parameters, assumed small, which describe the departure from the simplest VEV alignment, the eigenvalues of the matrices are given as [22]

$$
\begin{aligned}
m_{u, d, e} & =0, \\
m_{c, s, \mu} & =\left|y_{2,4,6} k^{u, d}-y_{1,3,5} v^{u, d}\right|, \\
m_{t, b, \tau} & =\left|y_{2,4,6} k^{u, d}+y_{1,3,5} v^{u, d}\right| .
\end{aligned}
$$

When one takes into account the small perturbations, $\epsilon_{i}$, one finds that the golden formula

$$
\frac{m_{\tau}}{\sqrt{m_{e} m_{\mu}}} \approx \frac{m_{b}}{\sqrt{m_{d} m_{s}}} .
$$

This successful formula nicely relates down-type quark and charged lepton masses. On the other hand, the doubled Higgs structure forced by PQ symmetry allows us to avoid the unwanted top quark mass prediction $\frac{m_{\tau}}{\sqrt{m_{e} m_{\mu}}} \approx \frac{m_{t}}{\sqrt{m_{u} m_{c}}}$ present in [22]. Let us note that the golden formula relating quark and lepton masses in Eq. (10) also emerges in other flavor symmetry schemes, such as the ones proposed in [13-15], but without connection to an underlying PecceiQuinn symmetry.

\section{EMERGENCE OF THE CKM MATRIX}

We now show that, in addition to Eq. (10), our scheme provides a dynamical framework for the CKM matrix describing quark mixing and $C P$ violation.

It is clear from Eqs. (6), (7), and (8) that, in the limit of vanishing $\epsilon_{i}$, the charged fermions of the first family are massless. Moreover, when the perturbations $\epsilon_{i} \rightarrow 0$, the matrix that diagonalizes $M_{u, d} \cdot M_{u, d}^{\dagger}$ is given by

$$
V_{L}^{u, d}=\left(\begin{array}{ccc}
1 & 0 & 0 \\
0 & 1 / \sqrt{2} & 1 / \sqrt{2} \\
0 & -1 / \sqrt{2} & 1 / \sqrt{2}
\end{array}\right),
$$

for up- and down-type quarks, with eigenvalues given by Eq. (9). This means that the CKM matrix, defined as $V_{\mathrm{CKM}}=V_{L}^{u} V_{L}^{d \dagger}$, is naturally "aligned" to be just the identity matrix.

The perturbations of the eigenvectors of $M \cdot M^{\dagger}$ which result from turning on the perturbations around the minima get translated into a small shift of the matrices in Eq. (11), which no longer coincide. Their mismatch is the CKM matrix. After turning on these perturbations, the electron and the up and down quarks, all get nonzero masses, while small quark mixing angles emerge naturally.

Thanks to the structured breaking of the $S O(3)$ family symmetry, one can predict mixing angles in terms of quark masses. We have the well-known Gatto-Sartori-Tonin [27] relation for the Cabibbo angle

$$
\theta_{C} \approx \sqrt{\frac{m_{d}}{m_{s}}}-\sqrt{\frac{m_{u}}{m_{c}}}
$$

while for $\left|V_{u b}\right|$ we get

$$
\left|V_{u b}\right| \approx \frac{\sqrt{m_{d} m_{s}}}{m_{b}}-\frac{\sqrt{m_{u} m_{c}}}{m_{t}},
$$


which extends a relation found in Ref. [22]. Finally, the doubling of scalar quintuplets $\left\langle\Phi^{u, d}\right\rangle$ plays a crucial role in generating $\left|V_{c b}\right|$, given as

$$
\left|V_{c b}\right|=\frac{\epsilon_{1}^{u}}{2 k^{u}}-\frac{\epsilon_{1}^{d}}{2 k^{d}}
$$

In contrast to $\theta_{C}$ and $\left|V_{u b}\right|$, the $\left|V_{c b}\right|$ matrix element can only emerge from the duplicated set of quintuplets, i.e., from the fact that $\Phi^{u}$ and $\Phi^{d}$ are different fields. Otherwise, the $b$ quark would decay predominantly to up quarks through the weak charged current. Thus, in the present framework, mass hierarchies and mixing angles arise as perturbations around the symmetry breaking minima of the scalar potential, rather than hierarchies in the Yukawa couplings. $C P$ violation can be accommodated through nontrivial phases in the Yukawa couplings, but no useful prediction emerges.

In short, the predictions we found confirm the expectation from a simple parameter counting. Including Yukawa couplings and VEVs we have in total 10 relevant parameters to describe 13 observables, namely the 9 charged fermion masses plus the 4 CKM parameters. ${ }^{2}$ This leads to three successful relations. Two of these are the predictions for the charged fermion masses, Eq. (10), and for the Cabibbo angle, Eq. (12). The third relation is given in Eq. (13). Notice that, thanks to the PQ symmetry, the golden relation in Eq. (10) is a successful one, as it involves only the down-type fermions, in contrast to Ref. [22]. Likewise, we have that $\left|V_{c b}\right|,\left|V_{u b}\right| \neq 0$, as required. Note also the important role played by the scalar potential dynamics, namely, the need for VEV misalignment in Eq. (3).

\section{NEUTRINO MASSES AND MIXINGS}

Neutrino masses arise naturally in $S O(10)$ unification through a conventional (type I) seesaw mechanism [8] [16-21]. In order to capture this at our level of analysis we add right-handed neutrinos $\nu_{R}$ transforming under the Peccei-Quinn symmetry, as in Table I.

The relevant Yukawa Lagrangian to generate neutrino masses is given by

$$
\mathcal{L}_{\nu}=\bar{l}_{L}\left(y_{D} \Psi^{u}+\tilde{y}_{D} \Phi^{u}\right) \nu_{R}+\bar{\nu}_{R}^{c}\left(y_{M} \sigma+y_{M}^{\prime} \rho\right) \nu_{R} .
$$

The vacuum expectation values of $\rho$ and $\sigma$ break the PecceiQuinn symmetry spontaneously, as well as lepton number. The last terms in Eq. (15) generate Majorana masses for $\nu_{R}$ after symmetry breaking. Notice also that $\langle\sigma\rangle$ breaks the $S O(3)$ family symmetry.

\footnotetext{
${ }^{2}$ The neutrino sector is discussed separately, see below.
}

To support a viable seesaw mechanism, both $\rho$ and $\sigma$ are necessary. If there were only the flavor singlet, neutrino mixing would be similar to that of quarks, hence small, and ruled out by the neutrino oscillation data [28]. Were there only the quintuplet, a two index traceless symmetric tensor, the seesaw would be singular, leaving four light neutrinos, instead of three. The symmetry breaking pattern obtained through the simultaneous presence of $\sigma$ and $\rho$ plays a key role in order to account for why neutrinos mix in such a different way from quarks.

In short, in our model the quark mixing and $C P$ violation arise from departures from the simplest VEV alignment of the Higgs fields, $\epsilon_{i} \neq 0$ in Eq. (3), and are significantly constrained. In contrast, neutrino masses and (generically large) lepton mixing are directly associated with PecceiQuinn breaking.

\section{HIGGS SCALAR SPECTRUM}

Our explicit implementation of $S O(3)$ flavor symmetry requires several scalar multiplets. In the context of renormalizable quantum field theory, without further constraints, there are many scalar coupling terms, andgiven that most of the spectrum is lifted to a high mass scale-few observational handles on them. Thus a complete analysis is both impractical and pointless, but we do need to ensure that an acceptable low-energy sector can emerge.

Generically, all the fields other than the axion will acquire mass terms of order the flavor and PQ breaking scale, barring cancellations between bare and induced mass terms. For purposes of $S U(2) \times U(1)$ breaking, we require at least one much lighter doublet. Notoriously, that requires a conspiracy or fine-tuning among parameters. This is an aspect of the hierarchy problem, which we do not address here. The only slight good news is that the existence of more than one doublet would require additional finetuning, so that the minimal one doublet structure, which so far is supported by experimental observations, is minimally unnatural.

To illustrate the mechanism whereby induced mass terms arise, consider the quartic operator $\Psi_{u}^{\dagger} \Psi_{u} \sigma^{\dagger} \rho$. Its contraction is unique and can be easily visualized in matrix form. If $\langle\sigma\rangle$ is aligned in the diagonal (recall it is symmetric and traceless) and $\rho$ is an $S O(3)$ singlet we get, after $S O(3)$ breaking takes place,

$$
\begin{aligned}
& \langle\rho\rangle \Psi_{u}^{\dagger}\left\langle\sigma^{\dagger}\right\rangle \Psi_{u} \\
& \quad=v_{\rho}\left(\begin{array}{lll}
\Psi_{1 u}^{\dagger} & \Psi_{2 u}^{\dagger} & \Psi_{3 u}^{\dagger}
\end{array}\right)\left(\begin{array}{ccc}
0 & 0 & 0 \\
0 & -v_{\sigma} & 0 \\
0 & 0 & v_{\sigma}
\end{array}\right)\left(\begin{array}{l}
\Psi_{1 u} \\
\Psi_{2 u} \\
\Psi_{3 u}
\end{array}\right) \\
& \quad=-v_{\sigma} v_{\rho}\left|\Psi_{2 u}\right|^{2}+v_{\sigma} v_{\rho}\left|\Psi_{3 u}\right|^{2} .
\end{aligned}
$$


One sees that the vacuum expectation value of the above operator generates a splitting of order the flavor/PQ-breaking scale among the electroweak doublet components of $\Psi_{u}$, so that two of them can be made heavy, i.e., at the large symmetry breaking scale, leaving the other massless. This argument may be escalated to the full scalar potential, which contains many relevant quartics, viz.

$$
\begin{array}{llllll}
\Phi_{u}^{\dagger} \Phi_{u} \sigma^{\dagger} \sigma, & \Phi_{u}^{\dagger} \Phi_{u} \sigma^{\dagger} \rho, & \Phi_{u}^{\dagger} \Phi_{u} \rho^{\dagger} \rho, & \Psi_{u}^{\dagger} \Psi_{u} \sigma^{\dagger} \sigma, & \Psi_{u}^{\dagger} \Psi_{u} \sigma^{\dagger} \rho, & \Psi_{u}^{\dagger} \Psi_{u} \rho^{\dagger} \rho, \\
\Phi_{d}^{\dagger} \Phi_{d} \sigma^{\dagger} \sigma, & \Phi_{d}^{\dagger} \Phi_{d} \sigma^{\dagger} \rho, & \Phi_{d}^{\dagger} \Phi_{d} \rho^{\dagger} \rho, & \Psi_{d}^{\dagger} \Psi_{d} \sigma^{\dagger} \sigma, & \Psi_{d}^{\dagger} \Psi_{d} \sigma^{\dagger} \rho, & \Psi_{d}^{\dagger} \Psi_{d} \rho^{\dagger} \rho, \\
\Phi_{u}^{\dagger} \Psi_{u} \sigma^{\dagger} \sigma, & \Phi_{u}^{\dagger} \Psi_{u} \sigma^{\dagger} \rho, & \Phi_{u}^{\dagger} \Psi_{u} \sigma \rho^{\dagger}, & \Phi_{d} \Psi_{d}^{\dagger} \sigma^{\dagger} \sigma, & \Phi_{d} \Psi_{d}^{\dagger} \sigma^{\dagger} \rho, & \Phi_{d} \Psi_{d}^{\dagger} \sigma \rho^{\dagger}, \\
\Phi_{u} \Phi_{d} \sigma^{\dagger} \sigma^{\dagger}, & \Phi_{u} \Phi_{d} \sigma^{\dagger} \rho^{\dagger}, & \Phi_{u} \Phi_{d} \rho^{\dagger} \rho^{\dagger}, & \Psi_{u}^{\dagger} \Psi_{d}^{\dagger} \sigma \sigma, & \Psi_{u}^{\dagger} \Psi_{d}^{\dagger} \sigma \rho, & \Psi_{u}^{\dagger} \Psi_{d}^{\dagger} \rho \rho, \\
\Phi_{d} \Psi_{u} \sigma^{\dagger} \sigma^{\dagger}, & \Phi_{d} \Psi_{u} \sigma^{\dagger} \rho^{\dagger}, & \Phi_{u}^{\dagger} \Psi_{d}^{\dagger} \sigma \sigma, & \Phi_{u}^{\dagger} \Psi_{d}^{\dagger} \sigma \rho . & &
\end{array}
$$

(These can be obtained in a systematic way; see [29].) One finds that, after breaking, the scalar mass ${ }^{2}$ matrix typically contains suitable off-diagonal terms, ensuring that the light doublet is a linear combination of $\Psi^{u}, \Psi^{d}, \Phi^{u}, \Phi^{d}$ wherein each appears with a nonzero coefficient.

\section{DISCUSSION}

Before closing, we comment briefly on three issues which deserve mention.

(1) The PQ symmetry $U(1)_{\mathrm{PQ}}$ is conserved at the classical level, and to all orders in perturbation theory, but violated nonperturbatively. One can visualize the breaking using QCD instantons, and infer its character by analyzing anomalies. In this way, one may discover that a nontrivial $Z_{N}$ subgroup of $U(1)_{\mathrm{PQ}}$ is valid even nonperturbatively. Our model, as it stands, has $N=12$, with doubly charged scalar fields. If scalar fields which are not $Z_{N}$ singlets acquire VEVs, the possibility of domain walls arises. Such domain walls are very dangerous for early universe cosmology [30]. The most straightforward way to avoid this difficulty is to assume that the $Z_{N}$ breaking is followed by a period of cosmic inflation, so that potential domain walls get pushed beyond the horizon. Another possibility is to arrange that $N=1$. (We could also allow $N=2$, since the PQ breaking VEVs have PQ charge 2). This does not occur in our model as it stands, but it can be achieved by adding suitable colored fermions. In the absence of other motivations, however, that construction seems contrived.

(2) The vacuum expectation values of the $\rho$ and $\sigma$ scalars are responsible both for Peccei-Quinn and lepton number symmetry breaking. This entails an interesting conceptual relation between the axion and neutrino mass scales of the form

$$
m_{a} \sim\left(\Lambda_{\mathrm{QCD}} m_{\pi} / v^{2}\right) m_{\nu},
$$

where $m_{\pi}$ is the pion mass and $v$ is the electroweak scale. This relation, which implies that the axion mass is parametrically smaller than the neutrino mass, according to the square of the ratio of QCD to electroweak scales. Since it assumes that the Yukawa couplings involving the neutrino field is of order unity, it should be applied using the heaviest of the light neutrinos. Of course, we cannot preclude the possibility that PQ symmetry breaks at a higher scale, through condensates which do not generate neutrino masses; this effect could drive the axion mass down further.

(3) The presence of extra gauge bosons coupled to flavor will mediate $\Delta F=2$ neutral flavor changing interactions at tree level. The most sensitive probe appears to be $K^{0}-\bar{K}^{0}$ mixing [31]. From this we estimate

$$
\frac{g^{2}}{M_{F}^{2}} \lesssim \frac{1}{\left[10^{4} \mathrm{TeV}\right]^{2}}
$$

where the gauge boson mass is $M_{F} \sim g f_{a}$. This constrains the Peccei-Quinn breaking scale to be $f_{a} \gtrsim 10^{7} \mathrm{GeV}$, a much weaker bound than arises from astrophysical constraints [32].

\section{SUMMARY}

Motivated by ideas arising in comprehensive unification based on spinors, we have considered possible consequences of supplementing the Standard Model gauge symmetry with commuting $S O(3)$ flavor and PQ symmetries in a way consistent with $S O(10)$ embedding. Proceeding in a bottom-up way, we analyzed a minimal $S O(3)_{F} \times U(1)_{\mathrm{PQ}}$ extension of the Standard Model unifying the three families of matter. Fairly simple choices of multiplet structure and symmetry breaking pattern allowed us to accommodate the known phenomenology of quark and lepton masses and mixings and to make several nontrivial connections among them. The PQ symmetry 
was important to this success, and of course it continues to serve its familiar roles in ensuring accurate strong $T$ symmetry and in providing, in axions, a good dark matter candidate.

\section{ACKNOWLEDGMENTS}

This work is funded by the Spanish Grants No. SEV2014-0398 and No. FPA2017-85216-P (AEI/FEDER, UE) and PROMETEO/2018/165 (Generalitat Valenciana). The work of M. R. is supported by FPU Grant No. FPU16/ 01907. F. W.'s work is supported by the U.S. Department of Energy under Contract No. DE-SC0012567, by the European Research Council under Grant No. 742104, and by the Swedish Research Council under Contract No. 335-2014-7424. M. R. would like to thank Aqeel Ahmed for helpful conversations.
[1] R. D. Peccei and H. R. Quinn, $C P$ Conservation in the Presence of Instantons, Phys. Rev. Lett. 38, 1440 (1977).

[2] S. Weinberg, A New Light Boson?, Phys. Rev. Lett. 40, 223 (1978).

[3] F. Wilczek, Problem of Strong $p$ and $t$ Invariance in the Presence of Instantons, Phys. Rev. Lett. 40, 279 (1978).

[4] G. Bertone, D. Hooper, and J. Silk, Particle dark matter: Evidence, candidates and constraints, Phys. Rep. 405, 279 (2005).

[5] J. W. F. Valle and J. C. Romao, Neutrinos in High Energy and Astroparticle Physics (John Wiley \& Sons, New York, 2015).

[6] L. M. Krauss and F. Wilczek, Discrete Gauge Symmetry in Continuum Theories, Phys. Rev. Lett. 62, 1221 (1989).

[7] I. de Medeiros Varzielas, G. G. Ross, and J. Talbert, A unified model of quarks and leptons with a universal texture zero, J. High Energy Phys. 03 (2018) 007.

[8] M. Gell-Mann, P. Ramond, and R. Slansky, Complex spinors and unified theories, Conf. Proc. C 790927, 315 (1979).

[9] F. Wilczek and A. Zee, Families from spinors, Phys. Rev. D 25, 553 (1982).

[10] J. Bagger and S. Dimopoulos, O(18) revived: Splitting the spinor, Nucl. Phys. B244, 247 (1984).

[11] G. F. Giudice and S. Raby, A new paradigm for the revival of technicolor theories, Nucl. Phys. B368, 221 (1992).

[12] M. Reig, J. W. F. Valle, C. A. Vaquera-Araujo, and F. Wilczek, A model of comprehensive unification, Phys. Lett. B 774, 667 (2017).

[13] S. Morisi, E. Peinado, Y. Shimizu, and J. W. F. Valle, Relating quarks and leptons without grand-unification, Phys. Rev. D 84, 036003 (2011).

[14] S. Morisi, M. Nebot, K. M. Patel, E. Peinado, and J. W. F. Valle, Quark-lepton mass relation and CKM mixing in an $A 4$ extension of the minimal supersymmetric standard model, Phys. Rev. D 88, 036001 (2013).

[15] C. Bonilla, S. Morisi, E. Peinado, and J. W. F. Valle, Relating quarks and leptons with the $T_{7}$ flavour group, Phys. Lett. B 742, 99 (2015).

[16] P. Minkowski, $\mu \rightarrow e \gamma$ at a rate of one out of 1-billion muon decays?, Phys. Lett. 67B, 421 (1977).

[17] T. Yanagida, Horizontal symmetry and masses of neutrinos, Conf. Proc. C 7902131, 95 (1979).

[18] S. Glashow, The future of elementary particle physics, in Proceedings of the 1979 Cargese Summer Institute on Quarks and Leptons, Vol. 687, edited by J.-L. Basdevant,
D. Speiser, J. Weyers, R. Gastmans, and M. Jacob (Plenum Press, New York, 1980).

[19] R. N. Mohapatra and G. Senjanovic, Neutrino Mass and Spontaneous Parity Nonconservation, Phys. Rev. Lett. 44, 912 (1980).

[20] J. Schechter and J. W. F. Valle, Neutrino masses in $S U(2) x U(1)$ theories, Phys. Rev. D 22, 2227 (1980).

[21] G. Lazarides, Q. Shafi, and C. Wetterich, Proton lifetime and fermion masses in an $S O(10)$ model, Nucl. Phys. B181, 287 (1981).

[22] F. Wilczek and A. Zee, Horizontal Interaction and Weak Mixing Angles, Phys. Rev. Lett. 42, 421 (1979).

[23] S. F. King and G. G. Ross, Fermion masses and mixing angles from $S U(3)$ family symmetry and unification, Phys. Lett. B 574, 239 (2003).

[24] Y. Ema, K. Hamaguchi, T. Moroi, and K. Nakayama, Flaxion: A minimal extension to solve puzzles in the standard model, J. High Energy Phys. 01 (2017) 096.

[25] L. Calibbi, F. Goertz, D. Redigolo, R. Ziegler, and J. Zupan, Minimal axion model from flavor, Phys. Rev. D 95, 095009 (2017).

[26] M. Dine, W. Fischler, and M. Srednicki, A simple solution to the strong $C P$ problem with a harmless axion, Phys. Lett. 104B, 199 (1981).

[27] R. Gatto, G. Sartori, and M. Tonin, Weak selfmasses, Cabibbo angle, and broken $S U(2) \times S U(2)$, Phys. Lett. 28B, 128 (1968).

[28] P. F. de Salas, D. V. Forero, C. A. Ternes, M. Tórtola, and J.W. F. Valle, Status of neutrino oscillations 2018: First hint for normal mass ordering and improved $C P$ sensitivity, Phys. Lett. B 782, 633 (2018).

[29] R. M. Fonseca, The Sym2Int program: Going from symmetries to interactions, J. Phys. Conf. Ser. 873, 012045 (2017).

[30] Ya. B. Zeldovich, I. Yu. Kobzarev, and L. B. Okun, Cosmological consequences of the spontaneous breakdown of discrete symmetry, Zh. Eksp. Teor. Fiz. 67, 3 (1974) [Sov. Phys. JETP 40, 1 (1974)].

[31] C. Patrignani et al. (Particle Data Group), Review of particle physics, Chin. Phys. C 40, 100001 (2016).

[32] M. M. Miller Bertolami, B. E. Melendez, L. G. Althaus, and J. Isern, Revisiting the axion bounds from the Galactic white dwarf luminosity function, J. Cosmol. Astropart. Phys. 10 (2014) 069. 